\title{
Breast cancer-related lymphedema: focus on surgical treatment
}

\author{
Maria Luisa Nardulli \\ Department of Plastic Surgery and Burns, Breast Reconstruction Unit, Perrino Hospital, Brindisi 72100, Italy.
}

Correspondence to: Dr. Maria Luisa Nardulli, Department of Plastic Surgery and Burns, Breast Reconstruction Unit, Perrino Hospital, Brindisi 72100, Italy. E-mail: marialuisanardulli@gmail.com

How to cite this article: Nardulli ML. Breast cancer-related lymphedema: focus on surgical treatment. Plast Aesthet Res 2020;7:15. http://dx.doi.org/10.20517/2347-9264.2019.56

Received: 20 Nov 2019 First Decision: 11 Feb 2020 Revised: 5 Mar 2020 Accepted: 10 Mar 2020 Published: 31 Mar 2020

Science Editor: Xiao Long Copy Editor: Jing-Wen Zhang Production Editor: Tian Zhang

\begin{abstract}
Breast cancer-related lymphedema (BCRL) can affect breast cancer patients, especially after axillary surgery and radiation treatment, for life. First line treatment is conservative and involves physical therapy and compression. It requires absolute, life-long compliance with treatment by the patient and, in some cases, it is ineffective. In recent years, surgery has emerged as a possible alternative or even, complementary therapy for BCRL. The most commonly reported techniques are reconstructive or debulking procedures. Reconstructive procedures are aimed at restoring the lymphatic pathways and can be effective early in the disease process, when increased arm volumes are mostly due to the accumulation of protein-rich fluid in the interstitial space. In more advanced stages, where fibrotic and hypertrophic adipose tissues are dominant, debulking techniques such as liposuction can be recommended. A standard of care for the treatment of BCRL has not been established. Currently, different techniques can be combined to optimize clinical outcomes, and the surgical approach must be individualized for each patient, based on sound clinical and imaging assessment. BCRL surgical treatment remains a challenging topic that requires further study before it can be standardized.
\end{abstract}

Keywords: Breast cancer-related lymphedema, vascularized lymph node transfer, lymphaticovenous anastomosis, liposuction, fat grafting

\section{INTRODUCTION}

Breast cancer-related lymphedema (BCRL) is a well-known, potential sequela of breast cancer treatment, which can result in damage to and impairment of the lymphatic drainage system of the upper limb.

cC) (C) The Author(s) 2020. Open Access This article is licensed under a Creative Commons Attribution 4.0 International License (https://creativecommons.org/licenses/by/4.0/), which permits unrestricted use, sharing, adaptation, distribution and reproduction in any medium or format, for any purpose, even commercially, as long as you give appropriate credit to the original author(s) and the source, provide a link to the Creative Commons license, and indicate if changes were made.

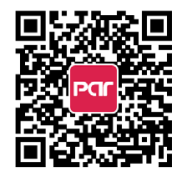


Consequently, protein-rich fluid accumulates in the interstitial space, leading to abnormal swelling and volume increase of the $\operatorname{arm}^{[1,2]}$. In more advanced stages, lymphedema is characterized by adipose deposition and irreversible fibrosis, together with residual lymphatic vessel disruption ${ }^{[3]}$. Despite reported incidence of BCRL being variable, DiSipio et al. ${ }^{[4]}$ referred the overall estimated incidence of upper limb lymhedema after breast cancer to be $21.4 \%$.

Symptoms of BCRL include arm heaviness, pain, impaired mobility, and recurrent skin and subcutaneous tissue infections. BCRL also affects the patient's body image with consequent psychological impairment ${ }^{[1,2,5]}$. For this reason, it is one of the most disabling sequelae of breast cancer treatment.

Risk factors for BCRL can be non-treatment or treatment related. Non-treatment related risk factors include $\mathrm{BMI}>30 \mathrm{~kg} / \mathrm{m}^{2[6,7]}$, cellulitis ${ }^{[8]}$ and genetic predisposition ${ }^{[9,10]}$. In fact, several studies have suggested that an underlying anomaly and/or dysregulation of the lymphatic system can lead to subclinical lymphatic dysfunction. In turn, this would increase the risk of developing lymphedema after disruption of the lymphatic network from surgery and/or radiation treatment. Treatment-related risk factors include axillary surgery, mastectomy and lack of breast reconstruction, regional lymph node radiation, and chemotherapy ${ }^{[1]}$. One of the main treatment-related risk factors for BCRL is axillary lymph node dissection (ALND) ${ }^{[1]}$ as it involves axillary clearance and thus, iatrogenic lymphatic damage. In a meta-analysis, DiSipio et al. ${ }^{[4]}$ reported the incidence of BCRL in patients with unilateral breast cancer at 19.9\% after ALND. On the contrary, sentinel lymph node biopsy, an alternative procedure to ALND in clinically node negative breast cancer patients, is reported to be associated with a four times lower incidence of BCRL compared to $\mathrm{ALND}^{[4,11]}$. This is likely because sentinel lymph node biopsy is less invasive. On the other hand, several studies have shown an increasing incidence of BCRL with the number of axillary nodes removed ${ }^{[12,13]}$.

Interestingly, mastectomy itself has been reported to be a risk factor for $\mathrm{BCRL}^{[1,4,12]}$. Regarding breast reconstruction, some studies suggest that delayed autologous breast reconstruction can improve existing lymphedema ${ }^{[14]}$. Card et al. ${ }^{[11]}$ concluded in a 6-year study that patients undergoing breast reconstruction after mastectomy had a lower risk of, and later onset of BCRL compared to patients who had undergone mastectomy alone. Card et al. ${ }^{[11]}$ also found no difference in the risk of BCRL between the different types of breast reconstruction (tissue expander/implant, latissimus dorsi and implant, free autologous abdominal tissue only). The authors thus hypothesized that the transfer of vascularized tissue onto the chest reduces scarring, bridges damaged lymphatic vessels and promotes angiogenesis ${ }^{[11,14]}$. With regard to expander/ implant based breast reconstruction, tissue expansion and capsule formation seem to increase the expression of vascular endothelial growth factor (VEGF), which has an important role in angiogenesis and lymphangiogenesis ${ }^{[10]}$, via chronic ischemia ${ }^{[15]}$.

Regional lymph node radiation (supraclavicular, with or without posterior axillary boost) is an independent risk factor for BCRL. It also conveys an increased risk of developing BCRL compared to breast/chest radiotherapy alone ${ }^{[16]}$.

In terms of the role of adjuvant and neoadjuvant chemotherapy in increasing BCRL risk, a literature review by Gillespie et al. ${ }^{[1]}$ [Figure 1] suggested that the current evidence was not conclusive because several studies have chemotherapy as a potential risk factor for BCRL but data from others were non-confirmatory.

Several staging systems have been proposed for lymphedema. One of the most widely used is that of the International Society of Lymphology (ISL), as shown in Table $1^{[17]}$. Early or mild stages can be characterized by a positive "pitting" test, when pressure exerted to an edematous limb by the thumb for at least 60 seconds induces a depression or indentation on the skin. Such indentation is attributable to the displacement of interstitial fluid. In more advanced stages, when adipose hypertrophy is dominant in the affected limb, edema is typically "non-pitting" ${ }^{[18]}$. 

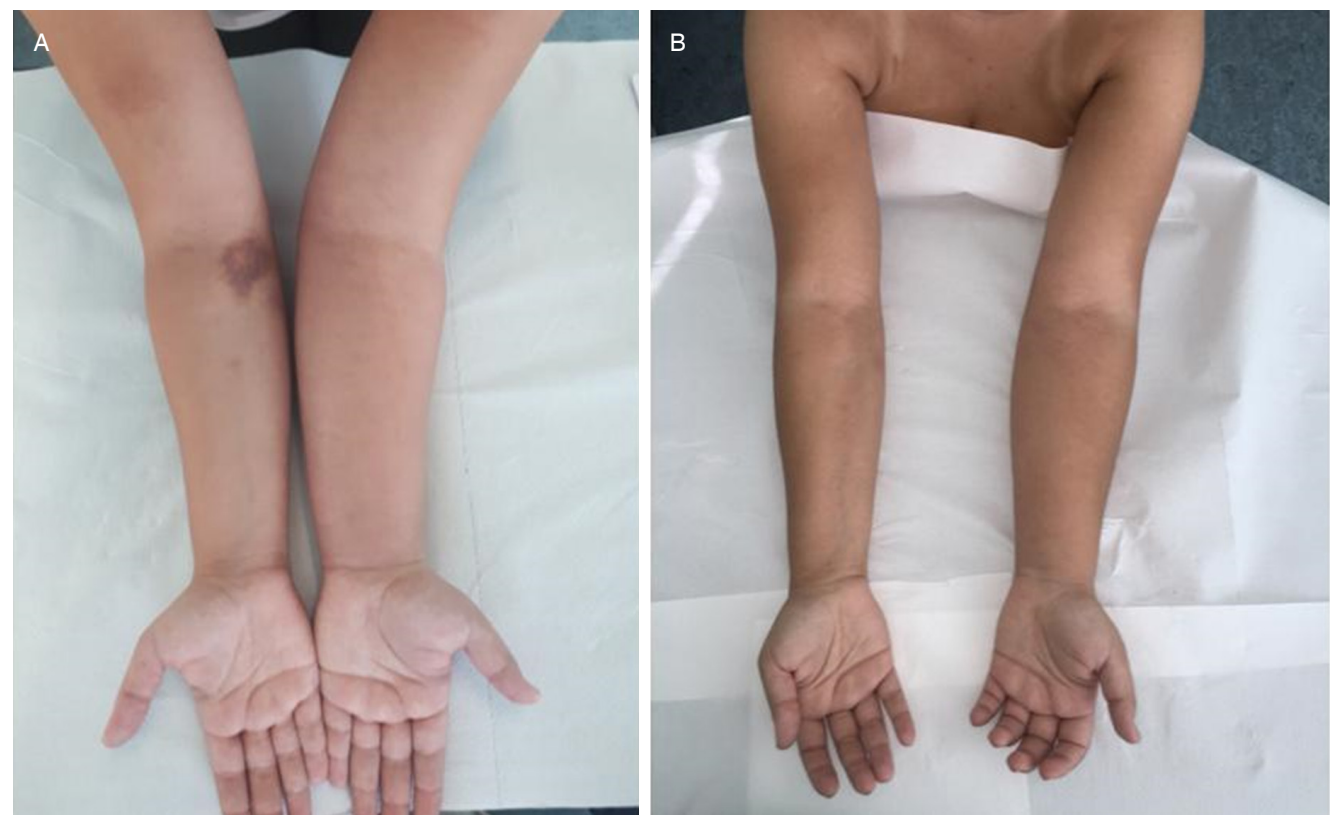

Figure 1. A: A patient who developed left upper limb lymphedema during neoadjuvant therapy with Paclitaxel for breast cancer; B: After undergoing left nipple sparing mastectomy, sentinel lymph node biopsy and immediate breast reconstruction with a prepectoral implant, the lymphedema improved spontaneously, especially at the level of the forearm

\section{Table 1. Staging system for lymphedema}

\begin{tabular}{ll}
\hline Stage O (or Ia) & $\begin{array}{l}\text { which refers to a latent or sub-clinical condition where swelling is not yet evident despite impaired lymph transport, subtle } \\
\text { changes in tissue fluid/composition, and changes in subjective symptoms. It may exist months or years before overt edema } \\
\text { occurs (Stages I-III) } \\
\text { represents an early accumulation of fluid relatively high in protein content (e.g., in comparison with "venous" edema) which } \\
\text { subsides with limb elevation. Pitting may occur. An increase in various proliferating cells may also be seen } \\
\text { Stage I } \\
\text { signifies that limb elevation alone rarely reduces tissue swelling and pitting is manifest. Late in Stage II, the limb may not pit as } \\
\text { excess subcutaneous fat and fibrosis supervenes } \\
\text { encompasses lymphostatic elephantiasis where pitting can be absent and trophic skin changes such as acanthosis, alterations } \\
\text { in skin character and thickness, further deposition of fat and fibrosis, and warty overgrowths have developed }\end{array}$ \\
Stage III &
\end{tabular}

This table summarizes the lymphedema staging system according to ISL ${ }^{[17]}$, from the 2016 Consensus Document of the ISL ${ }^{[17]}$ (adapted). These Stages only refer to the physical condition of the extremities. ISL: International Society of Lymphology

The first-line treatment for BCRL is a set of nonsurgical and conservative measures known as Complex Decongestive Therapy (CDT). CDT includes lymphatic manual drainage, bandaging, skin care, exercise, patient education and is coupled with the use of compression garments. The aim of CDT is to reduce limb volume first, followed by maintenance of the results achieved. Normally, patients suffering from lymphedema require lifelong adherence to such therapeutic measures ${ }^{[1,19,20]}$. Consequently, non-compliance to conservative therapy is frequent. Nevertheless, in recent years, interest in lymphedema surgery has increased all over the world and there have been many advances in both surgical techniques and imaging modalities. Therefore, surgery can be offered as a complementary or alternative therapeutic strategy for lymphedema, when conservative measures are inadequate ${ }^{[2,21]}$.

Below, we present an overview of the main surgical techniques currently adopted for the treatment of BCRL.

\section{SURGICAL TREATMENT FOR BCRL}

Surgical techniques for BCRL can be classified as physiological or reconstructive, and ablative or debulking procedures $^{[2,20]}$. Physiological or reconstructive techniques aim to restore lymphatic pathways in the axilla 
and upper limb to drain excess fluid accumulating in the arm by creating bypasses of lymph flow, or are based on the concept of inducing lymphangiogenesis ${ }^{[21]}$. Such techniques can be utilized in the early stages of BCRL, when a residual and functional lymphatic system can still be identified. "Pitting" edema is often present because excess limb volume is mainly caused by the accumulation of interstitial fluid. When edema progresses, the affected limb is characterized by increased tissue fibrosis, hypertrophy of adipose tissue, and irreversible damage and obliteration of the lymphatic vessels, thereby becoming "non-pitting". In such cases, physiological or reconstructive techniques are widely considered futile because the upper limb lymphatics are fibrotic and damaged. Moreover, the excess in limb volume is also attributed to fibrosis and fat hypertrophy instead. In these advanced, "non-pitting" stages, ablative or debulking procedures can reduce the excess volume of skin and/or subcutaneous tissue. In turn, this facilitates hygiene and improves limb functionality ${ }^{[2,19-21]}$.

\section{Ablative or debulking surgical techniques}

Ablative techniques aim to remove excess limb bulk to reduce lymphedema-associated morbidity. The Charles ${ }^{[22]}$ procedure was one of the earliest ablative techniques described. It involves the excision of excess skin and subcutaneous tissue up to the deep fascia, followed by skin grafting. However, this technique leads to extensive scarring, poor cosmetic results and the disruption of residual lymphatic vessels in the treated area. Therefore, it may even result in exacerbation of lymphedema. For these reasons, the Charles procedure is currently reserved for very advanced cases of lymphedema that are not susceptible to improvement through other measures ${ }^{[2,21]}$.

Excess fat and fibrotic tissue in the lymphadematous upper limb can now be removed through suction-assisted lipectomy. The technique was first applied to lymphedema treatment by O'Brien et al. ${ }^{[23]}$. Brorson et al. ${ }^{[24,25]}$ then popularized a technique consisting of large volume lipoaspiration in a limb affected by lymphedema ${ }^{[24-26]}$. These procedures are typically indicated in advanced, "non-pitting" chronic edema that is non-responsive to conservative measures as explained above. Recently, Hoffner et al. ${ }^{[26]}$ reported the 5-year results after liposuction and postoperative controlled compression therapy (CCT) in a series of 105 patients suffering from "non-pitting" or minimal "pitting" BCRL. The study protocol consists of the reduction of excess arm volume through liposuction or power-assisted liposuction, from the wrist to the shoulder, while the hand is spared. Volume reduction was performed according to previously assessed, contralateral limb volume measurements. In more recent cases, the authors used a tourniquet in addition to tumescence with adrenaline and lidocaine to reduce blood loss during surgery. A sterilized custom-made compression garment is also put on the arm in the operating theatre, as soon as surgery progresses, to reduce both blood loss and postoperative edema. With this approach, the authors obtained complete reduction of excess volume within 3-6 months and sometimes earlier. As expected, a key point of this approach is CCT, based on the constant use of made-to-measure compressive garments after surgery and indefinitely thence on. Despite the favorable long-term results achieved, CCT remains the main limitation of the treatment and requires absolute patient compliance. On the other hand, such liposuction-based techniques allow arm volume reduction and skin retraction without the need for skin excision or recurrence ${ }^{[26]}$, thereby improving patients' quality of life ${ }^{[25]}$. A reduced incidence of infections after liposuction has also been reported and is linked to the improvement of skin blood flow after the reduction of excess arm volume ${ }^{[25]}$. Moreover, if liposuction does not restore the lymphatic pathways in the affected limb, further impairment of lymphatic transport capacity after liposuction has not been proven ${ }^{[24-26]}$.

\section{Reconstructive or physiological surgical techniques}

Vascularized lymph node transfer

In vascularized lymph node transfer (VLNT), the lymph nodes (LNs) are harvested as a vascularized free flap with a vascular pedicle. The flap contains donor LNs embedded within the surrounding fat, with or without a skin paddle ${ }^{[2,27]}$. The rationale for VLNT is based on the concept that axillary LNs, if surgically 
removed or damaged by radiotherapy for breast cancer treatment, can be replaced by autologous healthy LNs harvested from an untreated donor $\operatorname{sit}^{[27]}$. VLNT should therefore improve BCRL because new lymphatic connections are expected to form between new afferent and efferent lymphatics sprouting from the transferred LNs and residual lymphatics at the recipient site. VEGF-C secreted by transplanted LNs also seems to have an important role in this mechanism ${ }^{[28]}$. Additionally, VLNTs are believed to absorb lymph like a sponge, before redirecting it into the vascular network like a pump ${ }^{[19,29,30]}$.

This technique is especially appealing to patients who have undergone ALND (with or without radiation treatment) and with scarring in the axilla ${ }^{[21]}$. As donor LNs replace axillary LNs after ALND and/or radiotherapy, the most common recipient site is obviously the axilla ${ }^{[2,27]}$. Insetting of the flap in the axilla should be preceded by surgical release of scars as the scar itself may impede lymphatic flow ${ }^{[27]}$. Scar release would also provide a healthy environment for lymphangiogenesis. Other VLNT recipient sites have also been described, such as the wrist and elbow ${ }^{[30,31]}$, where distal insetting in the upper limb can take advantage of gravity for the flap to absorb excess fluid.

Donor LNs can be harvested from different sites with the groin being the most popular ${ }^{[2,27]}$. Other donor sites include the supraclavicular area, submental area, lateral thoracic area and intra-abdominal $\mathrm{LNs}^{[19]}$. In the groin, the LN-flap is supplied by the superficial circumflex iliac artery or superficial inferior epigastric artery. Scaglioni and Suami ${ }^{[32]}$ found that in cadavers, the superficial inguinal LNs can be divided into 3 subgroups: the abdominal, medial thigh and lateral thigh groups. The abdominal group is utilized in VLNT. The LNs to be taken are located above the inguinal ligament, and superficial and lateral to the common femoral vessels. The harvesting of LNs below the inguinal ligament, medial to the femoral vessels or deep to the fascia of the thigh must be avoided because these LNs drain the lower limb and iatrogenic lower limb lymphedema may result ${ }^{[2,33]}$. Data now show that $\mathrm{LN}$-flap harvesting is not a complication-free procedure and VLNT from the groin can lead to lower limb lymphedema ${ }^{[33]}$.

As such, reverse lymphatic mapping techniques are gaining popularity with VLNT procedures. These imaging techniques aim to identify the LNs that drain lymph from the donor limb, to avoid inadvertent harvest during VLNT. Indocyanine green (ICG)-lymphography has been shown to be useful for this purpose. ICG is injected intradermally into the foot web spaces, absorbed by lymphatic vessels and transported proximally to the LNs draining the lower limb. Lymphatic vessels and nodes can be visualized in real-time with a camera that captures the near-infrared fluorescence emitted from ICG so that the draining LNs can be spared ${ }^{[2]}$. Similarly, patent blu dye V can be injected intradermally at the level of the anterior superior iliac spine with the aim of identifying LNs draining the lower abdomen ${ }^{[2]}$.

\section{Lymphatico-venous anastomosis}

The lymphatico-venous anastomosis (LVA) [Figure 2] technique bypasses the lymphatic obstruction, creating a shunt between the lymphatic and blood circulation in the affected limb. The first LVA was performed several decades ago ${ }^{[34,35]}$, but the currently accepted technique was developed after the advent of supermicrosurgery ${ }^{[36]}$.

First, a small quantity of patent $\mathrm{V}$ blue dye is injected intradermally, a few centimeters distal to where the surgical incision will be performed. The dye is absorbed by lymphatic vessels which then turn blue and become easier to identify. Multiple anastomoses between superficial subcutaneous lymphatic vessels and venules are then performed, either end-to-end or end-to-side, in the affected upper limb. Lymph is thus diverted into the venous circulation ${ }^{[2]}$. This technique is widely accepted for the treatment of early BCRL, when lymphatic vessels with residual functionality can still be demonstrated, as lymph has to be pumped into venules through the LVA. ICG-lymphography and magnetic resonance lymphography can thus be useful for preoperative assessment for the presence of, and location of contractile lymphatics suitable for $\operatorname{LVA}^{[2]}$. 


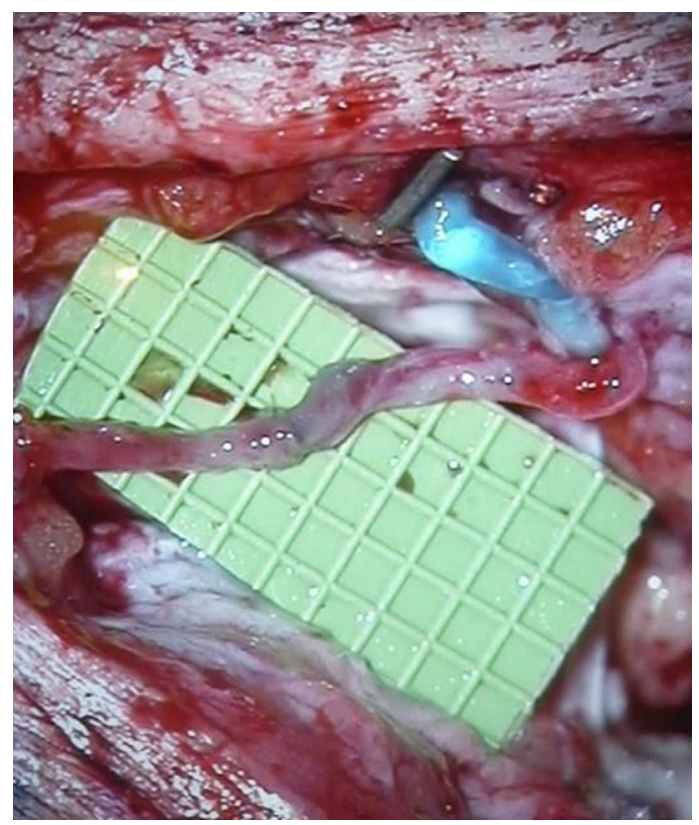

Figure 2. Magnified, intra-operative view of an lymphatico-venous anastomosis

\section{Lymphaticolymphatic bypass}

This procedure has been popularized by Baumeister et al. ${ }^{[37,38]}$. Healthy lymphatic vessels are harvested from the lower extremity, used as a graft, and are inset subcutaneously under the skin of the anterior shoulder, between the upper arm and the neck. The ends of the grafted lymphatic vessels are anastomosed to recipient lymphatic vessels in the arm and supraclavicular region. Despite lymphoscintigraphy demonstrating the patency of lymphatic vessels in the graft and a significant reduction of arm volume, this technique leads to a long scar at the donor site and the risk of lymphedema in the donor lower limb cannot be excluded ${ }^{[1,20]}$. In turn, Campisi et al ${ }^{[39]}$ described the use of an autologous vein graft in a similar manner to bypass the lymphatic obstruction, thereby sparing the lymphatic vessels in the donor site.

\section{Fat grafting}

Adipose-derived stem cells (ADSCs) are mesenchymal stem cells that can be collected through liposuction easily as fat tissue is an abundant and easily accessible source of ADSCs ${ }^{[40,41]}$. Recently, animal studies have demonstrated that the administration of ADSCs can increase the number of lymphatic vessels and improve secondary lymphedema ${ }^{[42]}$. The capacity of ADSCs to induce lymphaniogenesis seems to be mediated by VEGF-C and the release of other lymphangiogenetic factors. Saijo et al. ${ }^{[40]}$ studied the paracrine effects of ADSCs in promoting lymphangiogenesis in irradiated lymphatic endothelial cells in vitro. The results obtained suggest that ADSCs could have a role in the treatment of secondary-post irradiation limb lymphedema. Yet, few papers have described fat grafting in the axilla to improve BCRL clinically ${ }^{[41,43-45]}$. Maruccia et al. ${ }^{[45]}$ retrospectively compared the efficacy of upper limb circumference reduction and the improvement in patients' quality of life between VLNT alone and VLNT plus scar revision through fat grafting with better outcomes reported in the latter. Toyserkani et al. ${ }^{[43]}$ however, only reported a modest decrease in excess arm volume, that was not significant, after similar scar revision by means of fat grafting and ADSC injection in the axilla. Better results though were achieved in ISL stage I than in ISL stage II BCRL. The authors also observed an improvement in lymphedema symptoms and the decreased need for conservative treatment for the majority of patients. Recently, Toyserkani et al. ${ }^{[4]}$ reported similar results after a 1-year period of follow-up. Quantitative lymphoscintigraphy was used to evaluate upper limb lymph drainage after ADSC injection in the axilla but no significant improvement was observed. 

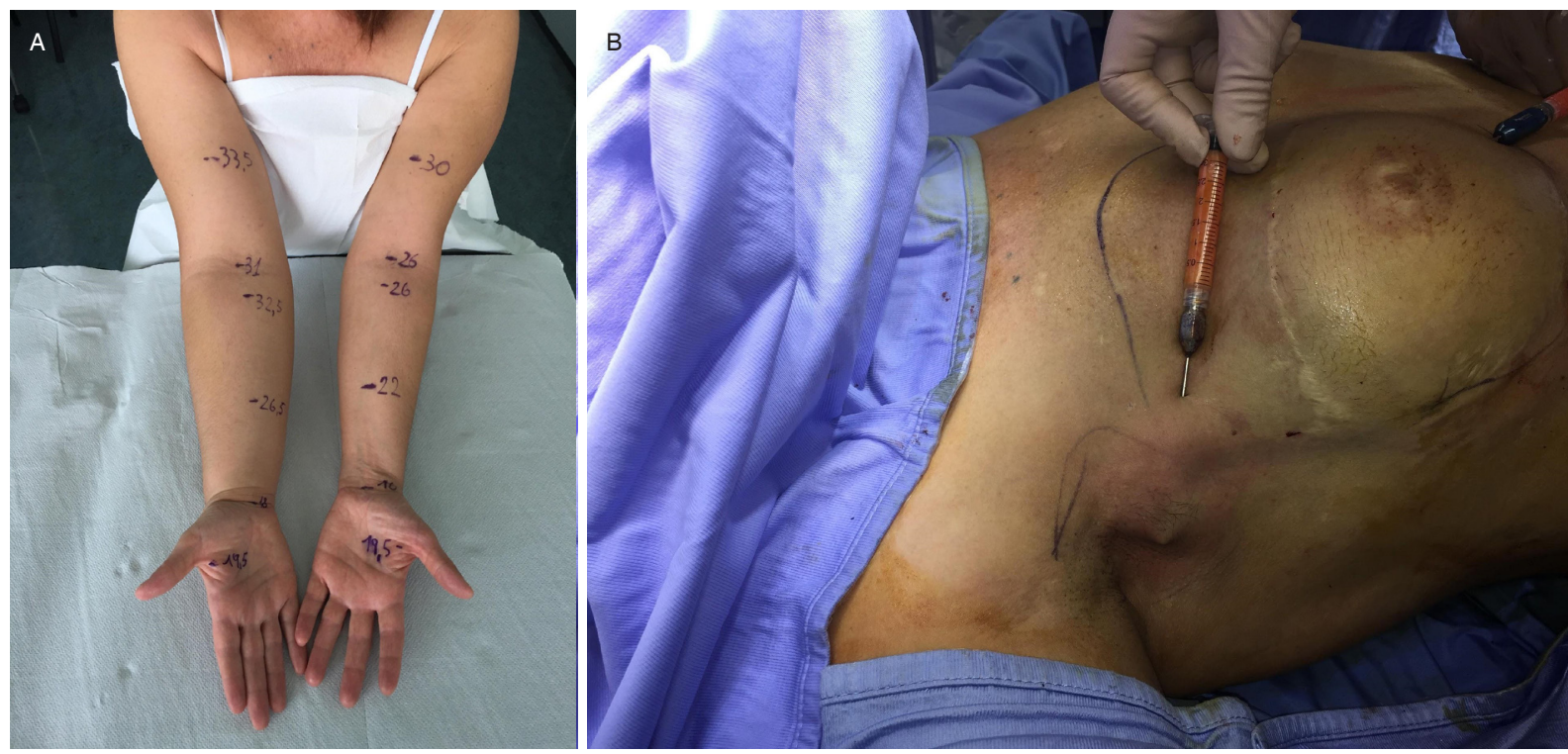

Figure 3. A: a patient with right breast cancer-related lymphedema, following axillary lymph node dissection and regional lymph node radiation. The patient had undergone right breast reconstruction with a TRAM-flap; B: in the same patient, fat grafting was carried out in the right breast for contour refinement and volume augmentation, and in the axilla for the release of fibrosis and filling of the residual defect from axillary lymph node dissection. TRAM: transverse rectus abdominis myocutaneous

The author of the present paper reports her preliminary experience in treating patients with BCRL by releasing fibrotic tissue in the axilla followed by fat grafting to the area [Figure $3 \mathrm{~A}$ and $\mathrm{B}$ ]. Five patients have been treated over 6 months. All patients underwent breast reconstruction and fat grafting. In addition to fat grafting of the breast, a small quantity (15 to $30 \mathrm{~mL}$ ) of fat was also transferred to the axilla, with the aim of releasing fibrotic tissue and filling up the dead space following axillary surgery. Fat harvest and injection were performed according to the standard Coleman technique ${ }^{[46,47]}$. Before fat injection, scar tissue in the axilla was released percutaneously ${ }^{[48]}$. Four patients had improvement in upper limb circumferences at 1 , 3 and 6 months follow-up. Furthermore, they reported subjective improvement in terms of reduction of arm heaviness and improved suppleness of the affected limb. No infections developed during the follow-up period. One patient however, reported a worsening in arm circumference volume and increasing heaviness after surgery.

This report does not claim to demonstrate the efficacy of fat grafting for BCRL. This is due to the small sample of patients treated, the short follow-up and the absence of significant results. These results though, show that the procedure is simple and quick, and appears to be safe, which is in line with previous studies. As fat grafting is often used in breast reconstruction, a small quantity of fat can easily be injected into the axilla for scar release. In vitro studies and animal models show that ADSCs induce lymphangiogenesis and could have a role in lymphedema treatment but there are few clinical studies on the same available. While the role of ADSCs in improving clinical BCRL requires further investigation, it is well accepted that axillary scar release can improve BCRL. This concept has also been highlighted in the context of VLNT ${ }^{[2,27]}$. Scar release through fat grafting should therefore be able to improve BCRL.

\section{Combined techniques and tailored treatment}

Currently, there is no gold standard for BCRL treatment but different surgical techniques can be combined to offer the patient a tailored approach based on staging and a global preoperative assessment. This is dependent on clinical examination and imaging modalities (e.g., ICG-lymphography, MR-lymphography, lymphoscintigraphy). As described earlier, non-pitting edema and the absence of residual functioning lymphatic vessels would necessitate an ablative procedure. Pitting edema in the advanced stages must be 
managed with CDT first. Early stage BCRL with pitting edema can benefit from reconstructive procedures. If functional lymphatic channels are available still, LVA can be considered. In patients with a scarred axilla, they may benefit from VLNT instead. Some authors combine VLNT and LVA with satisfactory results reported $^{[2,21]}$. VLNT from the groin can also be performed simultaneously with deep inferior epigastric perforator (DIEP)-flap breast reconstruction ${ }^{[49,50]}$ to address BCRL concurrently ${ }^{[2,49]}$.

Finally, laser-liposuction in combination with VLNT has been described for treating II stage (ISL staging) BCRL $^{[51]}$. Nicoli et al. ${ }^{[51]}$ described the use of laser liposuction in combination with VLNT to treat ten patients with stage II (ISL staging) BCRL who had failed a 6-month-period of conservative treatment. The twostage procedure involved VLNT from the supraclavicular or groin area to the wrist, and liposuction at 1 to 3 months later. Laser-assisted liposuction was carried out after tumescent solution infiltration, exsanguination and tourniquet positioning, using a high-power diode pulsed laser with 1470-nm wavelength. The laser light, conveyed through the microcannula, achieved both lipolysis and skin retraction in the affected arm. A traditional liposuction cannula was then used to aspirate the liquefied fat. Post-operatively, patients had to wear compressive garments at all times for the first 2 to 4 weeks and thereafter, only at night. Improvements in limb circumferences, skin tonicity and lymphoscintigraphic features in the treated arm have been reported by the authors. Histological changes including the reorganization of adipose cells and collagen in the reticular dermis have also been demonstrated in biopsies done post-procedure.

\section{CONCLUSION}

BCRL is a disabling sequela of breast cancer and associated treatments. A conservative approach (i.e., CDT) is the first line treatment for newly diagnosed BCRL. This treatment is insufficient in some cases, however, and these patients can benefit from surgical intervention depending on clinical and imaging assessment. To date, there remains no gold standard in the surgical treatment of BCRL. However, it is generally agreed that early stage BCRL can benefit from reconstructive procedures. Advanced stages with no or minimal pitting edema can be improved through liposuction. Each patient should therefore be assessed thoroughly before surgery and have a tailored treatment plan to maximize benefits. Newer strategies such as fat grafting and ADSC injection have shown promising preliminary results but must be investigated further. BCRL remains a highly challenging surgical problem.

\section{DECLARATIONS}

\section{Authors' contributions}

The author contributed solely to the article.

\section{Availability of data and materials}

Not applicable.

\section{Financial support and sponsorship}

None.

\section{Conflicts of interest}

There are no conflicts of interest.

\section{Ethical approval and consent to participate}

Not applicable.

\section{Consent for publication}

A written informed consent for using photos for scientific purposes was obtained by each patient. 


\section{Copyright}

(C) The Author(s) 2020.

\section{REFERENCES}

1. Gillespie TC, Sayegh HE, Brunelle CL, Daniell KM, Taghian AG. Breast cancer-related lymphedema: risk factors, precautionary measures, and treatments. Gland Surg 2018;7:379-403.

2. Masia J, Pons G, Nardulli ML. Combined surgical treatment in breast cancer-related lymphedema. J Reconstr Microsurg 2016;32:16-27.

3. Hespe GE, Nores GG, Huang JJ, Mehrara BJ. Pathophysiology of lymphedema-is there a chance for medication treatment? J SurgOncol 2017;115:96-8.

4. DiSipio T, Rye S, Newman B, Hayes S. Incidence of unilateral arm lymphoedema after breast cancer: a systematic review and metaanalysis. Lancet Oncol 2013;14:500-15.

5. De Brucker B, Zeltzer A, Seidenstuecker K, Hendrickx B, Adriaenssens N, et al. Breast cancer-related lymphedema: quality of life after lymph node transfer. Plast Reconstr Surg 2016;137:1673-80.

6. Jammallo LS, Miller CL, Singer M, Horick NK, Skolny MN, et al. Impact of body mass index and weight fluctuation on lymphedema risk in patients treated for breast cancer. Breast Cancer Res Treat 2013;142:59-67.

7. Ridner SH, Dietrich MS, Stewart BR, Armer JM. Body mass index and breast cancer treatment-related lymphedema. Support Care Cancer 2011;19:853-7.

8. Ferguson CM, Swaroop MN, Horick N, Skolny MN, Miller CL, et al. Impact of ipsilateral blood draws, injections, blood pressure measurements, and air travel on the risk of lymphedema for patients treated for breast cancer. J Clin Oncol 2016;34:691-8.

9. Hespe GE, Ly CL, Kataru RP, Mehrara BJ. Baseline lymphatic dysfunction amplifies the negative effects of lymphatic injury. Plast Reconstr Surg 2019;143:77e.

10. Newman B, Lose F, Kedda MA, Francois M, Ferguson K, et al. Possible genetic predisposition to lymphedema after breast cancer. Lymphat Res Biol 2012;10:2-13.

11. Card A, Crosby MA, Liu J, Lindstrom WA, Lucci A, et al. Reduced incidence of breast cancer-related lymphedema following mastectomy and breast reconstruction versus mastectomy alone. Plast Reconstr Surg 2012;130:1169-78.

12. Kilbreath SL, Refshauge KM, Beith JM, Ward LC, Ung OA, et al. Risk factors for lymphoedema in women with breast cancer: a large prospective cohort. Breast 2016;28:29-36.

13. Kim M, Kim SW, Lee SU, Lee NK, Jung SY, et al. A model to estimate the risk of breast cancer-related lymphedema: combinations of treatment-related factors of the number of dissected axillary nodes, adjuvant chemotherapy, and radiation therapy. Int J Radiat Oncol Biol Phys 2013;86:498-503.

14. Chang DW, Kim S. Breast reconstruction and lymphedema. Plast Reconstr Surg 2010;125:19-23.

15. Lantieri LA, Martin-Garcia N, Wechsler J, Mitrofanoff M, Raulo Y, et al. Vascular endothelial growth factor expression in expanded tissue: a possible mechanism of angiogenesis in tissue expansion. Plast Reconstr Surg 1998;101:392-8.

16. Warren LE, Miller CL, Horick N, Skolny MN, Jammallo LS, et al. The impact of radiation therapy on the risk of lymphedema after treatment for breast cancer: a prospective cohort study. Int J Radiat Oncol Biol Phys 2014;88:565-71.

17. Executive Committee. The diagnosis and treatment of peripheral lymphedema: 2016 consensus document of the international society of lymphology. Lymphology 2016;49:170-84.

18. Hansson E, Brorson H. Liposuction of lymphedema of the extremities. In: Shiffman MA, Di Giuseppe A, editors. Liposuction: principles and practice. Berlin Heidelberg: Springer-Verlag Berlin Heidelberg; 2016. pp. 721-33.

19. Chang DW, Masia J, Garza R 3rd, Skoracki R, Neligan PC. Lymphedema: surgical and medical therapy. Plast Reconstr Surg 2016;138:209S-18.

20. Suami H, Chang DW. Overview of surgical treatments for breast cancer-related lymphedema. Plast Reconstr Surg 2010;126:1853-63.

21. Kung TA, Champaneria MC, Maki JH, Neligan PC. Current concepts in the surgical management of lymphedema. Plast Reconstr Surg $2017 ; 139: 1003 \mathrm{e}-13 \mathrm{e}$.

22. Charles RH. Elephantiasis scroti. In: Latham A, English TC, editors. A system of treatment. Vol. 3. London: Churchill; 1912.

23. O'Brien BM, Khazanchi RK, Kumar PA, Dvir E, Pederson WC. Liposuction in the treatment of lymphoedema; a preliminary report. Br J Plast Surg 1989;42:530-3.

24. Brorson H, Svensson H, Norrgren K, Thorsson O. Liposuction reduces arm lymphedema without significantly altering the already impaired lymph transport. Lymphology 1998;31:156-72.

25. Brorson H. From lymph to fat: liposuction as a treatment for complete reduction of lymphedema. Int J Low Extrem Wounds 2012;11:10-9.

26. Hoffner M, Ohlin K, Svensson B, Manjer J, Hansson E, et al. Liposuction gives complete reduction of arm lymphedema following breast cancer treatment-a 5-year prospective study in 105 patients without recurrence. Plast Reconstr Surg Glob Open 2018;6:e1912.

27. Becker C, Assouad J, Riquet M, Hidden G. Postmastectomy lymphedema: long-term results following microsurgical lymph node transplantation. Ann Surg 2006;243:313-5.

28. Viitanen TP, Visuri MT, Hartiala P, Mäki MT, Seppänen MP, et al. Lymphatic vessel function and lymphatic growth factor secretion after microvascular lymph node transfer in lymphedema patients. Plast Reconstr Surg Glob Open 2013;1:1-9.

29. Schaverien MV, Coroneos CJ. Surgical treatment of lymphedema. Plast Reconstr Surg 2019;144:738-58.

30. Lin CH, Ali R, Chen SC, Wallace C, Chang YC, et al. Vascularized groin lymph node transfer using the wrist as a recipient site for management of postmastectomy upper extremity lymphedema. Plast Reconstr Surg 2009;123:1265-75

31. Cheng MH, Chen SC, Henry SL, Tan BK, Lin MC, et al. Vascularized groin lymph node flap transfer for postmastectomy upper limb lymphedema: flap anatomy, recipient sites, and outcomes. Plast Reconstr Surg 2013;131:1286-98.

32. Scaglioni MF, Suami H. Lymphatic anatomy of the inguinal region in aid of vascularized lymph node flap harvesting. J Plast Reconstr 
Aesthet Surg 2015;68:419-27

33. Pons G, Masia J, Loschi P, Nardulli ML, Duch J. A case of donor-site lymphoedema after lymph node-superficial circumflex iliac artery perforator flap transfer. J Plast Reconstr Aesthet Surg 2014;67:119-23.

34. Yamada Y. The studies on lymphatic venous anastomosis in lymphedema. Nagoya J Med Sci 1969;32:1-21

35. O'Brien BM, Shafiroff BB. Microlymphaticovenous and resectional surgery in obstructive lymphedema. World J Surg 1979;3:3-15,121-3.

36. Koshima I, Inagawa K, Urushibara K, Moriguchi T. Supermicrosurgical lymphaticovenular anastomosis for the treatment of lymphedema in the upper extremities. J Reconstr Microsurg 2000;16:437-42.

37. Baumeister RG, Siuda S. Treatment of lymphedemas by microsurgical lymphatic grafting: what is proved? Plast Reconstr Surg 1990;85:64-74.

38. Baumeister RG, Siuda S, Bohmert H, Moser E. A microsurgical method for reconstruction of interrupted lymphatic pathways: autologous lymph-vessel transplantation for treatment of lymphedemas. Scand J Plast Reconstr Surg 1986;20:141-6.

39. Campisi C. Use of autologous interposition vein graft in management of lymphedema: preliminary experimental and clinical observations. Lymphology 1991;24:71-6.

40. Saijo H, Suzuki K, Yoshimoto H, Imamura Y, Yamashita S, et al. Paracrine effects of adipose-derived stem cells promote lymphangiogenesis in irradiated lymphatic endothelial cells. Plast Reconstr Surg 2019;143:1189e-200e.

41. Toyserkani NM, Jensen CH, Sheikh SP, Sørensen JA. Cell-assisted lipotransfer using autologous adipose-derived stromal cells for alleviation of breast cancer-related lymphedema. Stem Cells Transl Med 2016;5:857-9

42. Hayashida K, Yoshida S, Yoshimoto H, Fujioka M, Saijo H, et al. Adipose-derived stem cells and vascularized lymph node transfers successfully treat mouse hindlimb secondary lymphedema by early reconnection of the lymphatic system and lymphangiogenesis. Plast Reconstr Surg 2017;139:639-51.

43. Toyserkani NM, Jensen CH, Andersen DC, Sheikh SP, Sørensen JA. Treatment of breast cancer-related lymphedema with adipose-derived regenerative cells and fat grafts: a feasibility and safety study. Stem Cells Transl Med 2017;6:1666-72.

44. Toyserkani NM, Jensen CH, Tabatabaeifar S, Jørgensen MG, Hvidsten S, et al. Adipose-derived regenerative cells and fat grafting for treating breast cancer-related lymphedema: lymphoscintigraphic evaluation with 1 year of follow-up. J Plast Reconstr Aesthet Surg 2019;72:71-7.

45. Maruccia M, Elia R, Ciudad P, Nacchiero E, Nicoli F, et al. Postmastectomy upper limb lymphedema: combined vascularized lymph node transfer and scar release with fat graft expedites surgical and patients' related outcomes. A retrospective comparative study. J Plast Reconstr Aesthet Surg 2019;72:892-901.

46. Coleman SR. Long-term survival of fat transplants: controlled demonstrations. Aesthetic Plast Surg 1995;19:421-5.

47. Coleman SR, Saboeiro AP. Fat grafting to the breast revisited: safety and efficacy. Plast Reconstr Surg 2007;119:775-85.

48. Khouri RK, Smit JM, Cardoso E, Pallua N, Lantieri L, et al. Percutaneous aponeurotomy and lipofilling: a regenerative alternative to flap reconstruction? Plast Reconstr Surg 2013;132:1280-90.

49. Saaristo AM, Niemi TS, Viitanen TP, Tervala TV, Hartiala P, et al. Microvascular breast reconstruction and lymph node transfer for postmastectomy lymphedema patients. Ann Surg 2012;255:468-73.

50. Chang EI, Masià J, Smith ML. Combining autologous breast reconstruction and vascularized lymph node transfer. Semin Plast Surg 2018;32:36-41.

51. Nicoli F, Constantinides J, Ciudad P, Sapountzis S, Kiranantawat K, et al. Free lymph node flap transfer and laser-assisted liposuction: a combined technique for the treatment of moderate upper limb lymphedema. Lasers Med Sci 2015;30:1377-85. 